Original article

\title{
Evaluation of obstetrical factors related to Sheehan syndrome
}

Takahiro Matsuwaki ${ }^{1}$, Khaleque Newaz Khan ${ }^{1 *}$, Tsuneo Inoue ${ }^{1}$, Atsushi Yoshida ${ }^{1}$, Hideaki Masuzaki ${ }^{1}$

a'Department of Obstetrics and Gynecology, Nagasaki University Graduate School of Biomedical Sciences, Nagasaki, Japan.

Running head: Clinical factors in Sheehan syndrome

*Address for correspondence:

Khaleque Newaz Khan, MD, PhD

Department of Obstetrics and Gynecology

Graduate School of Biomedical Sciences, Nagasaki University

1-7-1 Sakamoto, Nagasaki 852-8501, Japan

Tel: +81-95-819-7363; Fax: +81-95-819-7365

Email: nemokhan@nagasaki-u.ac.jp 


\begin{abstract}
Aim: To evaluate factors related to the occurrence of Sheehan syndrome.

Materials and Methods: The obstetrical DIC score, total volume of hemorrhage, shock index, level of consciousness at the time of shock occurrence, and pituitary MRI findings were evaluated in nine women who showed massive hemorrhage during delivery. These clinical outcomes were analyzed in all these patients who were prospectively followed-up to identify any possible occurrence of Sheehan syndrome.
\end{abstract}

Results: Comparing to six women with non-Sheehan syndrome, three women who were diagnosed with Sheehan syndrome showed significant elevation of the obstetrical DIC score, decrease in the level of consciousness during shock, and remarkable pituitary gland atrophic change with an empty sella turcica detected by pituitary MRI. The volume of hemorrhage during delivery and shock index were not significantly different between these two groups of women.

Conclusions: Careful attention and follow-up should be paid to women with postpartum massive hemorrhage in order to early detection and management of women with Sheehan syndrome. 
Key words: Sheehan syndrome, obstetrical outcome, MRI findings

\section{Introduction}

Sheehan syndrome is the name given to postpartum hypopituitarism and is caused by an infarction in the adenohypophysis, usually precipitated by massive uterine hemorrhage. Sheehan syndrome, an endocrine disease, was first reported in 1937 by the English pathologist Harold Leeming Sheehan on the basis of autopsy findings in patients with postpartum pituitary necrosis ${ }^{1-3}$. Postpartum massive hemorrhage is one of the causes of ischemia-induced pituitary dysfunction secondary to the arrest of blood flow to the adenohypophysis. Necrotized areas of the adenohypophysis undergo organization, form a fibrous scar leading to time-dependent atrophic change of pituitary gland resulting in empty sella turcica, the lodging bed of pituitary gland, which can be clinically detected by magnetic resonance imaging (MRI).

Clinically, women with Sheehan syndrome exhibited dysfunction of the anterior lobe of the pituitary gland with the manifestation of obstetrical shock accompanied by DIC during delivery. Decreased endocrine function can be restricted to one hormone or can affect all hormones secreted by adenohypophysis cells. Clinical 
presentation varies and is dependent on the age of the patient, rapidity of onset,

nature/causes of the pathological process, and the proportion of the affected

adenohypophysis cells. Functional impairment occasionally extended to the posterior

lobe of the pituitary.

While the incidence is low, being 1 out of 10,000 cases of delivery ${ }^{2}$, it is a

serious disease, and its pathological factors largely remain unclear. It is a hazardous

disease that may not only impair the quality of life of women but also may threaten life if

left untreated. Mild hypopituitarism can remain undetected for years but complete loss of

adenohypophysis function requires immediate treatment. Therefore, careful follow-up

intervention for early diagnosis and proper management of patients with Sheehan

syndrome is mandatory. However, information on the suitable clinical factors related to

the occurrence of Sheehan syndrome is scanty.

In our current study, we followed-up a group of women had suffered from

massive hemorrhage during delivery to identify the possible occurrence of Sheehan

syndrome. Secondly, we plan to analyze a number of obstetrical factors and hormonal

parameters in these women during the course of follow-up. 


\section{Subjects and Methods}

All subjects in this study were women of reproductive age who attended our

University hospital for screening and follow-up after achieving pregnancy. A total of nine women who suffered from massive hemorrhage during delivery was followed-up for a period of 13-80 months to identify any subsequent occurrence of Sheehan syndrome. Massive hemorrhage was defined as a loss of $\geq 3,000 \mathrm{~g}$ of blood including amniotic fluid. Mean age ranges from 29-41years and gestational week at delivery ranges from 31wks-41wks in these women. All women had at least one or more than one children.

During this follow-up period, any woman suspected to have Sheehan syndrome on the basis of clinical findings and hormonal profiles were referred to the Department of Internal Medicine of our University for final diagnosis by MRI.

Our current study is in accordance with the guidelines of the Declaration of

Helsinki and was performed with the approval by the Institutional Review Board of Nagasaki University. An informed consent was obtained from all participated women.

The obstetrical DIC score, total volume of hemorrhage, shock index (S.I.), level of consciousness, electrolyte levels, and pituitary MRI findings were evaluated in 
these women. The S.I. was shown as the maximum value during shock, and the level of consciousness was graded according to the criteria of Glasgow Coma Scale (GCS). ${ }^{4}$ The GCS criteria is based on a scoring system depending on eye response (score 1-4), verbal response (score 1-5) and motor response (score 1-6). A tendency towards higher GCS score indicates good consciousness level and a tendency towards decreased GCS score denotes bad consciousness level. The scoring system of consciousness level as measured by Japan Coma Scale (JCS) is somehow similar to that of GCS score.

Serum levels of hormones being secreted by the anterior lobe of the pituitary gland such as growth hormone (GH), luteinizing hormone ( $\mathrm{LH})$, follicle stimulating hormone (FSH), prolactin (PRL), thyroid stimulating hormone (TSH), and adrenocorticotropic hormone (ATCH) as well as estradiol (E2), insulin-like growth factor 1 (IGF-1), free thyroxine (FT4), and cortisol were measured by commercial based Immulyze assay.

All results are expressed as mean \pm SD. The clinical characteristics of the subjects were compared with one-way analysis of variance. Any difference of clinical factor or hormonal profile between two groups was analyzed by the non-parametric 
Mann-Whitney U-test or Student's t-test. Due to small number of cases in each group, we confirmed the validity of our results by Fisher's exact test. A value of $\mathrm{p}<0.05$ was considered statistically significant.

\section{Results}

During the follow-up period after postpartum massive hemorrhage, three cases were diagnosed as Sheehan syndrome (Sheehan group) and the remaining six cases did not develop Sheehan syndrome despite massive hemorrhage during delivery (non-Sheehan group). Obstetrical backgrounds of these two groups of cases are shown in Table 1. Symptoms suggestive of deficiency of pituitary anterior lobe hormones such as breast atrophy, poor milk secretion, hair loss, and malaise were observed from early in the puerperal period in the Sheehan group but not in the non-Sheehan group. The clinical backgrounds of three cases in the Sheehan group are described here briefly.

\section{CASE 1}

This patient (34 yrs old) was admitted to our department in January 2006 at 33 weeks of gestation due to pregnancy complicated by uterine myoma and threatened premature labor and underwent emergency Caesarean section due to placental abruption 
on the 5th hospital day. Uterine bleeding did not stop even after Caesarean section, and emergency supra-vaginal amputation of the uterus was carried out. Breast atrophy and hair loss of the head and pubic region were noted immediately after surgery. Estrogen replacement therapy was introduced 3 months after surgery due to decreased serum estradiol level. A diagnosis of Sheehan syndrome was made (LH and FSH: low response, PRL: low response, ACTH and cortisol: low response, TSH: normal response) based on the pituitary anterior lobe hormone stimulation tests [LH-RH test (LH, FSH), TRH test (TSH, PRL), CRH test (ACTH, cortisol), and GHRP-2 test (GH)] 4 years after surgery.

\section{CASE 2}

The patient (39 yrs old) with a history of previous Caesarean section underwent repeated Caesarean section again in November 2009 at 39 weeks of gestation and was referred to our department for emergency treatment due to marked adhesion in the abdominal cavity and failure to achieve complete hemostasis by suturing. After her arrival, emergency supra-vaginal amputation of the uterus was carried out, but arterial hemorrhage from the vaginal wall persisted, and hemostasis was eventually achieved by trans-catheter arterial embolization. After surgery, no milk secretion was noted, but hot 
flushes and mild hair loss were observed. She also became languid, showed little change in emotion, and gave the impression that her personality had changed. Six months after surgery, pituitary anterior lobe hormone stimulation tests were performed, and a diagnosis of Sheehan syndrome was made based on abnormal hormonal profiles (LH and FSH: low response, PRL: low response, ACTH, cortisol: low response, TSH: normal response, and marked growth hormone deficiency).

\section{CASE 3}

In June 2008, this patient (38 yrs old) underwent induction of labor using oxytocin for weak pain and subsequently an emergency Caesarean section was performed due to placental abruption. Uterine bleeding did not stop due to atonic bleeding even after abdominal closure, and the patient was transferred to our department for emergency treatment. After arrival, bleeding was stopped using a uterine contractor. No milk secretion was observed after surgery, amenorrhea persisted even 8 months after surgery, and the blood estradiol level was low. Therefore, HRT was initiated one year and five months after surgery. Easy fatigability, headache, dizziness, and loss of libido were also noted after surgery. Two years after surgery, pituitary anterior lobe hormone stimulation 
tests were performed, and a diagnosis of Sheehan syndrome was made by abnormal hormonal profiles (LH and FSH: low response, PRL: low response, TSH, ACTH, cortisol: normal response, and growth hormone deficiency).

Table 2 shows the obstetrical DIC score, total volume of hemorrhage, shock index (S.I.), status of consciousness level, and findings on pituitary MRI in 9 cases. Between Sheehan and non-Sheehan groups, the total volume of hemorrhage was 9143.3g $(4,500-11,500)$ vs. $6695 \mathrm{~g}(3,260-10,900)(\mathrm{p}=0.365)$, and S.I. was $3.03(2.8-3.3)$ vs. 2.55 (1.6-3.5) $(\mathrm{p}=0.311)$, respectively, showing no significant difference, but the mean obstetrical DIC score was 16.3 (15-18) vs. 9.5 (7-13) (p=0.004), respectively, being significantly higher in the Sheehan group than in the non-Sheehan group. On pituitary MRI, also, an empty sella was noted in one case and pituitary atrophy in two cases in the Sheehan group, but no pituitary abnormality was observed in the non-Sheehan group (Fig. 1). The mean GCS score was 5.3 in the Sheehan group and 14.8 in the non-Sheehan group. GCS score was significantly low in cases with Sheehan syndrome $(p=0.0009)$ than in non-Sheehan syndrome. The level of consciousness based on Japan Coma Scale was JCS 0 or I (being conscious) in all cases in the non-Sheehan group but JCS II-30 and/or JCS 
III-300 (arose with stimuli or on command) in all patients in the Sheehan group, showing a significant decrease in the level of consciousness in the later group (Fisher's exact test, $\mathrm{p}=0.01)$.

Table 3 shows hormonal profiles in the sera of patients in the Sheehan group and non-Sheehan group. A decreased level of all measured hormones (except ACTH) was observed in patients in the Sheehan group when compared with cases in the non-Sheehan group. Although not statistically significant, serum GH/FSH levels were decreased to almost half and LH level was decreased to one third in cases of Sheehan group comparing to similar hormones levels in cases of non-Sheehan group (Table 3). Serum E2 level was undetectable (below cut off value, $<10 \mathrm{pg} / \mathrm{ml}$ ) in all cases with Sheehan syndrome in contrast to cases in the non-Sheehan group, Therefore, we could not analyze statistical difference of E2 levels between these two groups (Table 3). A statistically significant increase in basal hormone levels of ACTH ( $\mathrm{p}=0.011)$ and decrease in levels of IGF-1 $(p=0.048)$ was observed in the Sheehan group than in non-Sheehan group. Two cases of Sheehan syndrome with low cortisol level are now under treatment with cortical by doctor of Internal medicine in order to optimize the level of ACTH to the level of 
non-Sheehan group. Another case with Sheehan syndrome was not indicated for cortisol

treatment because basal cortisol level in this case was similar to that in non-Sheehan

group. We also observed an increased tendency in TSH levels in sera $(\mathrm{p}=0.061)$ of

patients in the Sheehan group.

Although data not shown, we also measured a number of electrolyte levels in

the sera of patients in the Sheehan and Sheehan group. Serum levels of sodium were

markedly reduced to $124 \mathrm{mEq} / \mathrm{L}$ in two cases in the Sheehan group and levels of sodium

were within normal range in all cases in the non-Sheehan group (data not shown). After

correction for albumin, serum calcium level was still low in both groups without showing any difference between these two groups (data not shown).

\section{Discussion}

Sheehan syndrome is a permanent secretion disorder of all pituitary anterior

lobe hormones caused by extensive pituitary necrosis secondary to obstetrical shock

accompanied by DIC following massive hemorrhage during delivery. No pituitary

dysfunction develops if about $50 \%$ of the normal pituitary gland remains intact, ${ }^{3}$ but

partial or panhypopituitarism is reported to occur if $70-90 \%$ of the pituitary gland is 
necrosed. ${ }^{5}$ Depending on the site and size of the necrosed area of the pituitary gland, the disease varies from panhypopituitarism (Sheehan syndrome), in which the secretion of all gonadal, thyroid, and adrenocortical hormones is reduced, to partial hypopituitarism, in which the secretion of 2 or more hormones is reduced, and to single-hormone hypopituitarism, in which the secretion of only one hormone is reduced. In our current study of nine cases with massive post-partum hemorrhage, we could detect three cases with Sheehan syndrome after combined and careful follow-up of these patients by Obstetrics doctors and doctors from Internal Medicine. All these three cases showed necrotic pituitary gland on MRI image and significant decrease in serum levels of at least two hormones (estradiol/IGF-1) when compared with cases in the non-Sheehan group. In fact, we found a decreased tendency of all pituitary anterior lobe, gonadal and thyroid hormones (except ACTH) in cases with Sheehan syndrome. Among all measured hormone levels in sera, we found significantly increase/decrease in the basal levels of ACTH/IGF-1, respectively and an increased tendency in the levels of TSH in cases in the Sheehan group than in non-Sheehan group. All other decreased or undetectable hormone levels in Sheehan group failed to achieve a significant difference 
comparing to non-Sheehan group. This could be due to small number of cases in each group.

There is a mutual feed-back loop between ACTH and cortisol. The minimal decrease of cortisol level in Sheehan group (Table 3) stimulates the secretion of ACTH in a positive feed-back mechanism in an attempt to compensate the damage of anterior pituitary lobe. This may explain the significantly increased basal ACTH levels in three cases with Sheehan syndrome comparing to cases in the non-Sheehan group. Two cases of Sheehan syndrome with low cortisol level are now under cortisol treatment by doctor of Internal medicine in order to normalize the level of ACTH to the level of non-Sheehan group. According to the comment of doctor of Internal medicine, basal cortisol level is important in the decision of cortisol treatment. Another case with Sheehan syndrome was not indicated for cortisol treatment, because basal cortisol level was similar to that of cases in the non-Sheehan group.

Besides reduction in hormone levels, we also found a significant increase in DIC score and significantly decreased levels of consciousness in patients with Sheehan syndrome. We could not find any significant difference in the total volume of hemorrhage 
or shock index between these two groups. This might be due to less number of cases in our current study.

It has been reported that deficiency of milk secretion is a frequent initial symptom of Sheehan syndrome, that symptoms such as breast atrophy, sense of fatigue, loss of pubic and axillary hair, and continuation of amenorrhea, and that hypothyroidism and hypoadrenalism are observed for several months to a few years. Sert et al. reported insufficiency of milk secretion in 26 of 28 patients diagnosed with Sheehan syndrome, ${ }^{6}$ and Kelestimur mentioned insufficiency of milk secretion as one of the diagnostic criteria for Sheehan syndrome. ${ }^{7}$ Of the 3 patients with Sheehan syndrome encountered at our department, insufficiency of milk secretion was observed in 2, and it is considered to be an important early puerperal symptom that may suggest Sheehan syndrome. Together with insufficient gonadotropin secretion, subsequent onset of breast atrophy and loss of pubic and axillary hair may be considered as an early indication of Sheehan syndrome as we experienced in our three cases.

Although GCS scoring system is internationally recognized to measure levels of consciousness, as an additional marker, we found in our current study that JCS was 
also equally useful for the evaluation of consciousness level. ${ }^{7}$ In fact, similar to decreased

GCS scores, we also found a score of JCS II-30 and/or JCS III-300 in all cases with

Sheehan syndrome. The decreased levels of consciousness in cases with Sheehan

syndrome can be explained by hyponatremia as we observed in two of our three cases.

Hyponatremia in patients with Sheehan syndrome may be secondary to suppression of

ADH secretion which could be a result of declining cortisol secretion. ${ }^{9,10}$ Sert et al.

measured serum sodium level, evaluated level of consciousness, and performed pituitary

MRI or CT in patients with Sheehan syndrome. They found a link between hyponatremia

and an empty sella in many patients exhibiting a reduced level of consciousness such as

coma and confusion. ${ }^{7}$ In our cases, abnormalities such as an empty sella were noted in all

patients in the Sheehan group by pituitary MRI, but no atrophic abnormality was

observed in the non-Sheehan group. Kaplun et al. reported a patient who showed massive

hemorrhage during delivery, a low-signal area on pituitary MRI on Day 26 after delivery,

and exhibited an empty sella 6 months after delivery. ${ }^{11}$ Therefore, serial pituitary MRI

from the early puerperal period may be considered necessary and recommended in

patients with massive hemorrhage during delivery in order to early identify and detect 
cases with Sheehan syndrome.

Sheehan syndrome is a rare but irreversible and serious disorder. When most pregnant women, who suffer massive hemorrhage or shock during delivery, survive and are rehabilitated, some patients are considered to have one-hormone hypopituitarism rather than panhypopituitarism such as typical Sheehan syndrome. However, publications on obstetrical predictive factors targeting to early identify or diagnose this debilitating disease are still inadequate. With strict prospective follow-up, here we demonstrated for the first time some subjective, hematological, hormonal and MRI imaging parameters which might be suitable in clinical practice to isolate and early diagnosis of Sheehan syndrome in groups of women had massive post-partum hemorrhage. In addition to subjective findings of insufficient or no milk secretion and breast atrophy, our current findings of decreased consciousness level and elevated obstetrical DIC score in women with massive post-partum hemorrhage may give us some additional clue in the isolation and early diagnosis of cases with Sheehan syndrome. Further study on this issue is necessary to strengthen our current findings.

Considering future QOL, early diagnosis of Sheehan syndrome based on our 
clinical findings is important for proper management of these patients by mutual

collaboration and discussion between Obstetrical doctors and doctors from Internal

Medicine. Basal cortisol and ACTH level may be an important parameter in decision

making for cortisol treatment in order to normalize ACTH level in cases with Sheehan

syndrome. Furthermore, careful attention and strict follow-up are mandatory for any

women with massive past-partum hemorrhage in order to search cases with Sheehan

syndrome.

Disclosure: The authors report no conflict of interest related to this paper.

\section{References}


1. Sheehan HL. Postpartum necrosis of the anterior pituitary. J Pathol Bacteriol

$1937 ; 45: 189-214$

2. Cunningham FG, Leveno KJ, Bloom SL, Hauth JC, Gilstrap III LC, Wenstrom KD.

McGRAW-HILL Publishing Division, Chapter 25, Obstetrical hemorrhage. Williams

Textbook of Obstetrics, p638, 21st edition:2001

3. Kovacs K. Sheehan syndrome. Lancet. 2003; 361:520-5224.

4. Teasdale G, Jennett B. Assessment of coma and impaired consciousness: a practical scale. Lancet 1974;13(2):81-84.

5. Ozbey N, Inanc S, Arai F, Azezli A, Orhan Y, Sencer E, Molvahilar S. Clinical and labolatory evaluation of 40 patients with Sheehan's syndrome. Ist J Med Sci $1994 ; 30: 826-829$

6. Sert M,Tetiker T, Kirim S, Kocak M. Clinical Report of 28 patients with Sheehan's Syndrome. Endocrine J 2003;50:297-301

7. Kelestimur F. Sheehan’s syndrome. Pituitary 2003;6:181-188

8. Ono K,Wada K, Takehara T, Shirotani T. Indication for Computed Tomography in Patients With Mild Head Injury. Neurol Med Chir (Tokyo) 2007;47:291 298 
9. Putterman C, Almog Y, Caraco Y, Gross DJ, Ben-Chetrit E. Inappropriate secretion of anti-diuretic hormone in Sheehan’s syndrome: A rare cause of postpartum hyponatremia. Am J Obstet Gynecol 1991;165:1330-1333

10. Wakui H, Nishimura T, Nishimura S, Endo Y, Nakamoto Y, Miura AB. Inapproporiate secretion of anti-diuretic hormone in isolated adrenocorticotropine deficiency. Am J Med Sci 1991;301:319-321

11. Kaplun J, Fratila C, Ferenczi A, Yang WC, Lantos G, Fleckman AM, Schubart UK. Sequential Pituitary MR Imaging in Sheehan Syndrome: Report of 2 cases. AJNR 2008;29:941-943 


\section{Figure legends}

Figure 1. Shows normal (left side) and abnormal (right side) pituitary gland by

T2 weighted image of magnetic resonance image (MRI). The red arrow and circle on the

left side shows iso-intensity to hyper-intensity area filling the sella turcica indicating

normal pituitary gland. The red arrow and circle on the right side shows hypo-intensity

area indicating necrotic pituitary gland causing the appearance of empty sella. 
Figure 1.

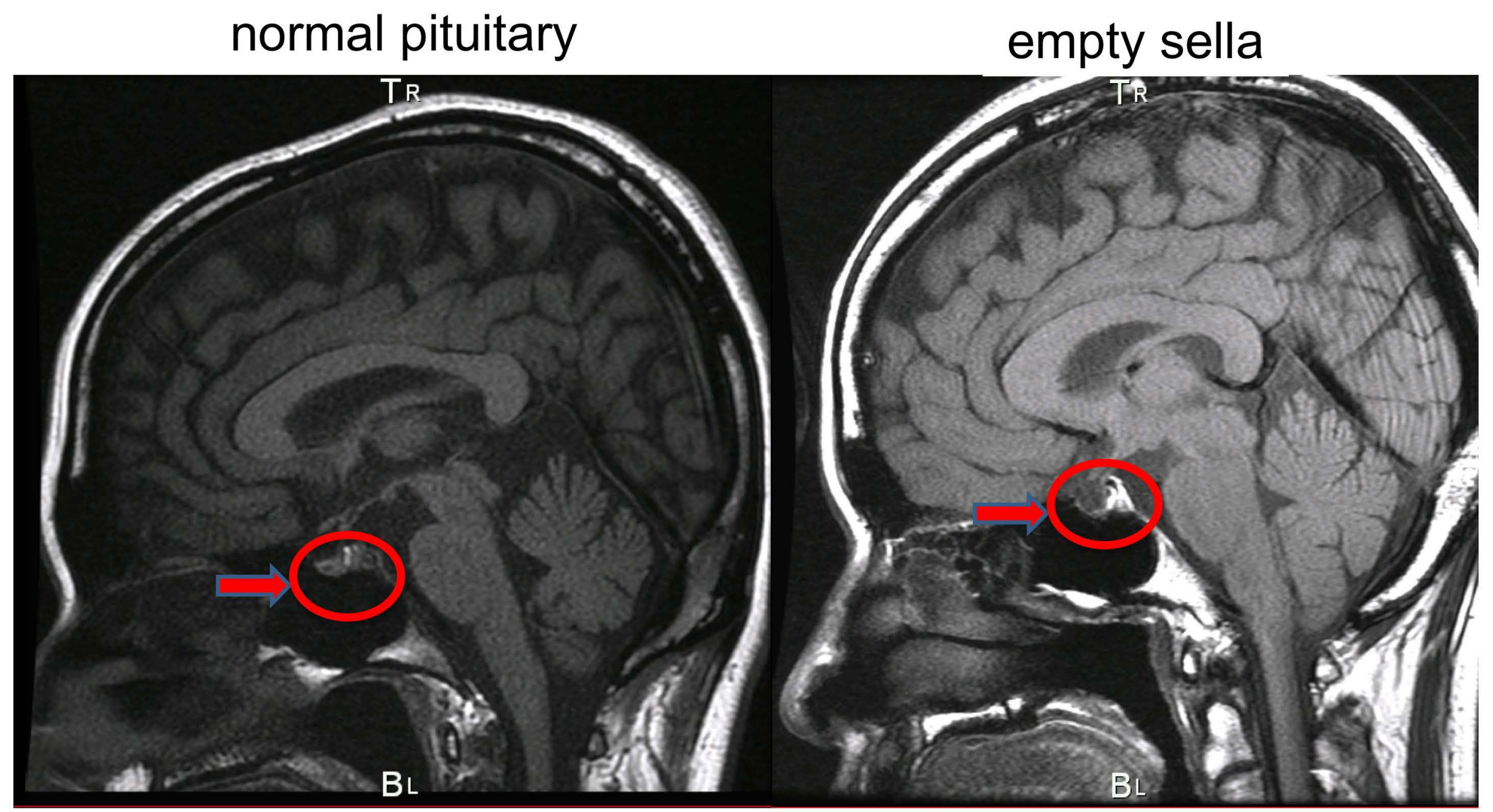


Table 1.Clinical profiles and obstetrical background of cases with and without Sheehan syndrome

\begin{tabular}{|c|c|c|}
\hline & Sheehan group $(n=3)$ & non-Sheehan group $(n=6)$ \\
\hline mean age in yrs. (range) & $41.7 \pm 0.6(41-42)$ & $32.2 \pm 1.9(29-34)$ \\
\hline gravida & $2.0 \pm 1.0$ & $2.17 \pm 1.6$ \\
\hline para & $2.0 \pm 1.0$ & $2.17 \pm 1.6$ \\
\hline $\begin{array}{l}\text { weeks of gestation at } \\
\text { delivery (range) }\end{array}$ & $37.7 \pm 3.2(34-40)$ & $37.7 \pm 3.9(31-41)$ \\
\hline mode of delivery & Caesarian section $(n=3)$ & $\begin{array}{l}\text { Caesarian section }(n=3) \\
\text { vaginal }(n=3)\end{array}$ \\
\hline cause of hemorrhage & $\begin{array}{l}\text { placental abruption }(n=2) \\
\text { insufficient suturing of uterine wall }(n=1)\end{array}$ & $\begin{array}{l}\text { uterine rupture }(n=1) \\
\text { atonic bleeding }(n=2) \\
\text { retroperitoneal hematoma }(n=2) \\
\text { placenta previa }(n=1)\end{array}$ \\
\hline $\begin{array}{l}\text { hysterectomy } \\
\text { symptom/sign }\end{array}$ & $\begin{array}{l}2 \text { cases }(66.7 \%) \\
\text { breast atrophy } \\
\text { lack of breast milk } \\
\text { hair loss } \\
\text { general fatigue } \\
\text { appetite loss }\end{array}$ & $\begin{array}{l}3 \text { cases }(50 \%) \\
\text { none }\end{array}$ \\
\hline $\begin{array}{l}\text { follow-up months after } \\
\text { delivery (range) }\end{array}$ & $51.3 \pm 28.5(23-80)$ & $19.5 \pm 5.9(13-27)$ \\
\hline
\end{tabular}

The results are expressed as mean $\pm \mathrm{SD}$. 
Table 2. Obstetrical outcome and image findings of cases with and without Sheehan syndrome

\begin{tabular}{llll}
\hline & Sheehan group $(\mathrm{n}=3)$ & non-Sheehan group $(\mathrm{n}=6)$ & P value \\
\hline DIC score & $16.3 \pm 1.5$ & $9.5 \pm 2.6$ & 0.004 \\
Total bleeding (g) & $9143.3 \pm 4021.4$ & $6695.0 \pm 3376.0$ & 0.365 \\
Shock Index & $3.03 \pm 0.25$ & $2.55 \pm 0.72$ & 0.311 \\
GCS score & $5.3 \pm 4.5$ & $14.8 \pm 0.4$ & 0.0009 \\
$\begin{array}{l}\text { Level of consciousness } \\
\text { based on JCS criteria }\end{array}$ & drowsy but can be aroused & can be aroused without & 0.01 \\
MRI of pituitary gland & empty sella $(\mathrm{n}=1)$ & stimuli (n=6) & \\
& atrophy $(\mathrm{n}=2)$ & normal $(\mathrm{n}=6)$ & \\
\hline
\end{tabular}

The results are expressed as mean \pm SD. Statistics were analyzed by Student's t-test or Fisher's

exact text. GCS, scores based on Glasgow Coma Scale; JCS, Japan Coma Scale 
Table 3. Hormonal profiles in sera of patients with and without Sheehan syndrome

\begin{tabular}{lllll}
\hline & normal value (range) & Sheehan group $(\mathrm{n}=3)$ & non-Sheehan group $(\mathrm{n}=6)$ & p value \\
\hline GH & $0.28 \sim 1.64 \mathrm{ng} / \mathrm{ml}$ & $0.93 \pm 0.46$ & $1.69 \pm 2.06$ & 0.235 \\
LH & $1.4 \sim 15 \mathrm{mIU} / \mathrm{ml}($ follicular phase) & $4.42 \pm 4.50$ & $13.31 \pm 16.30$ & 0.398 \\
& $0.5 \sim 15 \mathrm{mIU} / \mathrm{ml}($ luteal phase) & & & \\
FSH & $3 \sim 10 \mathrm{mIU} / \mathrm{ml}($ follicular phase) & $7.39 \pm 3.39$ & $15.62 \pm 25.46$ & 0.607 \\
& $1.3 \sim 6.2 \mathrm{mIU} / \mathrm{ml}($ luteal phase) & & & 0.842 \\
PRL & $5 \sim 30 \mathrm{ng} / \mathrm{ml}$ & $13.81 \pm 4.55$ & $15.13 \pm 10.25$ & 0.061 \\
TSH & $0.45 \sim 5.08 \mu \mathrm{IU} / \mathrm{ml}$ & $2.98 \pm 1.63$ & $1.50 \pm 0.41$ & 0.011 \\
ACTH & $7 \sim 56 \mathrm{pg} / \mathrm{ml}$ & $25.8 \pm 10.7$ & $10.9 \pm 2.8$ & not \\
Estradiol & $20 \sim 350 \mathrm{pg} / \mathrm{ml}($ follicular phase) & $\leqq 10.0($ less than cut off & $131.2 \pm 173.4$ & analyzed \\
& $45 \sim 300 \mathrm{pg} / \mathrm{ml}($ luteal phase) & value) & & 0.804 \\
Cortisol & $6.2 \sim 19.4 \mu \mathrm{g} /$ dl(morning) & $8.42 \pm 2.90$ & $9.04 \pm 3.56$ & 0.048 \\
& $2.3 \sim 12.3 \mu \mathrm{g} /$ dl(afternoon) & & & $149 \pm 50.8$ \\
IGF-1 & $73 \sim 311 \mathrm{ng} / \mathrm{ml}$ & $66.3 \pm 43.7$ & $1.19 \pm 0.17$ & 0.334 \\
Free $\mathrm{T}_{4}$ & $0.95 \sim 1.57 \mathrm{ng} / \mathrm{dl}$ & $1.06 \pm 0.18$ & &
\end{tabular}

The results are expressed as mean $\pm \mathrm{SD}$. GH, growth hormone; LH, luteunizing hormone;

FSH, follicle-stimulating hormone; PRL, prolactin; TSH, thyroid stimulating hormone;

ACTH, adrenocorticotropin hormone; IGF-1, insulin growth factor-1; free $\mathrm{T}_{4}$, free thyroxine 